\title{
Impacts of persistent general and site-specific pain on activities of daily living and physical performance: a prospective analysis of the English Longitudinal Study of Ageing
}

$\begin{array}{ll}\text { Dr Vasoontara Yiengprugsawan } & \text { vasoontara.yieng@anu.edu.au } \\ \text { Professor Andrew Steptoe } & \text { a.steptoe@ucl.ac.uk }\end{array}$

${ }^{1}$ Centre for Research on Ageing, Health and Wellbeing, Research School of Population Health, The Australian National University, Canberra, Australia.

2 Australian Research Council Centre of Excellence on Population Ageing Research 3

Department of Behavioural Science and Health, Institute of Epidemiology and Health Care, University College London, United Kingdom.

$\S$

Correspondence to: Dr Vasoontara Yiengprugsawan, The Australian National University, Research School of Population Health, Building 54 Florey, Acton 2601, Canberra, Australia Tel: +61 26125 56320; Email: vasoontara.yieng@anu.edu.au; vasoontara.yieng@gmail.com

Short running title: Longitudinal impacts of persistent pain 


\section{Abstract}

Aim: Pain is an increasingly common phenomenon as people age; pain over a long period can result in limited functioning. This study investigates the impacts of general and multi-site specific pain on activities of daily living and physical performance among older adults.

Methods: Data were analysed from the English Longitudinal Study of Ageing (ELSA), a representative sample of the population aged 50 years and older. Face-to-face interview and nurse records were used from Waves 2 to 6 (2004-2012) for analyses. General and site-specific pain (back, hip, and knee) were measured biennially between 2004 and $2008(n=5010)$. Impaired activity of daily living and physical performance measures (chair rise and grip strength) between 2008 and 2012 were aggregated across assessments as outcomes. Multinomial logistic regressions were used for the former and linear regression for the latter, adjusting for potential covariates.

Results: General pain (moderate to severe) was prevalent with close to a quarter of participants reporting pain at least twice during the follow-up period. Multi-site pain reports were strong predictors of subsequent limited activities of daily living (Adjusted Odds Ratios range from 1.86 to 3.97 for back and hip 2.04 to 4.19 for back and knee, and 2.08 to 5.16 for hip and knee). Persistent pain was also strongly associated with worse physical performance outcomes.

Conclusions: Our data confirm the longitudinal impacts of persistent pain among older adults. The findings highlight the value of monitoring and management of both general and site-specific pain in order to promote sustained independence at older ages.

Keywords: epidemiology; musculoskeletal disorders; pain management; physical function 


\section{Introduction}

Pain is an increasingly common phenomenon as people age. Intense pain can have a direct impact on higher levels of physical discomfort and limited mobility, and these issues may become severe among older people, affecting their quality of life and overall health (1-3).

Pain takes many forms and its impact may differ according to the duration, location, and severity. Musculoskeletal pain is common throughout the lifespan and has been identified as one of the top ten contributors to the global burden of diseases $(4,5)$. Chronic musculoskeletal pain was reported among adults in their 30 s and pain occurred frequently in the lower back, neck, shoulders, and knees (6). Among older persons, back, hip, and knee pain are the most common types (7-10). Longitudinal evidence, albeit quite limited, indicates that pain in the lower back and knees is associated with impaired physical function and quality of life among older Chinese (11).

Given the increasing proportion of older people in Western countries, the detection and management of avoidable effects of pain on daily life pose public health challenges. To investigate these impacts in the older population, we analysed data from the English Longitudinal Study of Ageing, in which frailty, mobility, and disability have previously been reported (12-14). We tested the hypothesis that repeated reports of pain over 3 waves of biennial data collection would predict impaired activities of daily living and poorer performance of upper and lower body actions over the subsequent 6 years.

We were particularly interested in investigating the persistence of pain using repeated measures over several years and the magnitude of locations of pain, including the overall level of pain, individual site specific (eg back, hip, and knee) as well as multisite pain. Longitudinal analyses reveal similarities and differences in patterns and gradients associated with the pain locations most affecting limitations of daily living and physical performance among older adults. 


\section{Materials and methods}

The English Longitudinal Study of Ageing (ELSA) is a cohort of men and women aged 50 and older living in England. It was set up in order to investigate the economic, social and health dynamics of ageing (15). ELSA began in 2002 with a face-to-face interview every two years. On alternate waves, participants are visited by a research nurse who carries out physical and biomarker assessments. We investigated longitudinal associations between pain status (Waves 2 to 4 ) and subsequent activities of daily living (Waves 4 to 6 ) and physical performance (data available in Waves 4 and 6). All analyses were based on 5,010 participants successfully followed up from Waves 2 (2004) to Wave 6 (2012).

Exposure variables: Participants were asked "Are you often troubled with pain?" "If yes, "how bad is the pain most of the time?" Responses were mild, moderate, or severe. Subsequently the respondents were asked about specific sites "In which parts of the body do you feel pain?" Multiple answers were allowed, including back, hips, and knees. For example, "how would you rate the pain in your back? "Where 0 is no pain and 10 is severe or excruciating pain, as bad as you can imagine'. ${ }^{1}$ Number of occasions across waves 2, 3 and 4 on which respondents rated their general pain as moderate/severe was counted, and individuals were categorised as never, once or twice. Similarly, site-specific pain rated as 5 and higher was classified as moderate/severe $(16,17)$, and the number of waves of pain reports was categorised (never, once, at least twice).

Activities of Daily Living and Instrumental Activities of Daily living (ADLs and IADLs): these measures have been widely used in international surveys (18). We identified five key $A D L$ and IADL outcomes to investigate associations with pain, including three items related to mobility: difficulty walking 100 yards; climbing several flights of stairs without rest; and

\footnotetext{
${ }^{1}$ Full English Longitudinal Study of Ageing questionnaire can be obtained from https://www.elsa-project.ac.uk
} 
stooping/ kneeling; and two sets of IADLs: dressing/showering and performing work around the house or garden. The number of limited ADLs/IADLs reported at least twice (Waves 4 to 6 ) were aggregated as $0-1,2-3$, and $4-5$.

Physical performance measures include: 1) chair stand test - a measure of lower body strength in which participants are asked to stand up and down as quickly as they can for five rises. The total length of time to complete the test was computed; 2) grip strength a measure of upper body strength in which the respondent is asked to squeeze a grip gauge up to three times with each hand. The average in kilograms for dominant and nondominant hands was combined (19).

The covariates in these analyses include age, sex, net total wealth income quintiles, long-standing conditions, and pain treatment. Net total wealth derived in ELSA includes housing and non-housing wealth (20). The former consists of primary house values subtract mortgage debt. The latter comprises gross financial wealth (eg savings, bonds, shares) minus financial debt (eg private loans, credit card), but also includes values of other property, business, and other assets (eg cars). Long-standing conditions are part of the selfperceived health module asking: "Do you have any long-standing illness, disability or infirmity...that has troubled you over a period of time, or that is likely to affect you over a period of time?" Pain treatment was assessed with question: "Are you currently receiving any treatment for your pain?" ${ }^{2}$ Analyses was also adjusted for Wave 2 body mass index known to be associated with physical functioning (21). We also included initial chair rise performance and initial grip strength as covariates in the analyses of chair rises and grip strength, respectively. A flow diagram of the study design is included as Figure 1.

\footnotetext{
${ }^{2}$ Derived ELSA financial variables and documentation can be assessed at http://doc.ukdataservice.ac.uk/doc/5050/mrdoc/pdf/5050 user guide financial derived vars.pdf Full English Longitudinal Study of Ageing questionnaire can be obtained from https://www.elsa-project.ac.uk
} 


\section{Statistical analysis}

Descriptive analyses reported prevalence of pain by sex and age at Wave 2 . We subsequently carried out multinomial logistic regressions on incident impaired ADLs/IADLs in Waves 4 to 6 categorised into $0-1,2-3$, and 4-5, excluding individuals who reported impaired ADLs/IADLs in Wave 2. The exposure variables were pain in Waves 2 to 4 and covariates as listed above. The analyses of objective physical performance, chair rise time and grip strength, averaged from Waves 4 and 6 involved multivariable linear regressions, with the same exposure measures as in the ADLs/IADLs analyses.

We tested three sets of association between pain and adverse outcomes. Model 1 assessed associations with general pain. Model 2 tested associations with each of the sitespecific pain locations (back, hip and knee). In Model 3, we tested three combinations of site-specific pain: back and hip, back and knee, and hip and knee. Results are presented as covariate-adjusted multinomial logistic odds ratios and multivariate regression coefficients with $95 \%$ confidence intervals.

\section{Results}

There were 5,010 respondents (2,818 men and 2,192 women) and their characteristics are summarised in Table 1. The prevalence of moderate/severe general pain increased with age from $16.5 \%$ to $23.5 \%$ among men and $23.0 \%$ to $38.0 \%$ among women (Figure 2), and was greater in women than men. Moderate/severe site-specific pain was also generally increased in prevalence with age, and was more common in women than men.

The frequency of general and site-specific pain (Waves 2 to 4) was reported in Table 2. A quarter of respondents reported moderate/severe general pain and $10.3 \%$, 
$6.6 \%$, and $9.9 \%$ reported back, knee, and hip pain at least twice across the three waves. Incident limitations in ADLs were aggregated across Waves 4, 5, and 6. Among those who never reported moderate/severe pain in Wave 2, 3, and 4, approximately 4\% reported incident limitations in walking, dressing/bathing, or performing work around the house in waves 4 to 6 , while difficulties climbing several flights of stairs and stooping/kneeling were more common (14\%-15\%).

The proportions reporting impaired ADLs/IADLs increased markedly for those reporting moderate/severe pain at least once or twice. (Table 2) For example, there was a five-fold increase (from 3.3\% to 19.5\%) in the prevalence of difficulty with walking 100 yards or dressing/bathing at follow-up in participants who reported moderate/severe pain at least twice between 2004 and 2008. Multi-site pain was reported at least twice for the combination of back and knee pain (4.9\%), back and hip pain (5.6\%), and knee and hip pain (4.4\%). The time taken for chair rises was longer and grip strength less for participants reporting persistent general pain and site-specific pain

After adjusting for covariates (Table 3), respondents reporting general and specific-site pain were 1.5 to 5 times more likely to report multiple impaired ADLs/IADLs on follow-up, compared with the no pain group. The effects were strongest for hip followed by knee and back pain. Persistent multi-site pain were strong predictors of subsequent adverse outcomes (Adjusted Odds Ratios range from 1.86 to 3.97 for back and hip 2.04 to 4.19 for back and knee, and 2.08 to 5.16 for hip and knee).

Pain during the exposure period was also associated with worse physical performance outcomes on follow-up. Persistent pain was associated with longer time taken to complete the chair rise tests in comparison with the no pain group, and there were stronger associations when pain was present more often, and from more sites of the 
body. For grip strength, results were statistically significant only for respondents who reported pain at least twice between Wave 2 (2004) and Wave 6 (2008).

\section{Discussion}

We set out to investigate the longitudinal impact of pain on impaired activities of daily living as well as objective measures of physical performance among older adults in England. Moderate to severe general pain reports were quite prevalent, with close to a quarter reporting pain at least twice across Waves 2, 3, and 4. Persistent reporting of multisite pain across the 4-year exposure period was particularly significant for future impaired activities of daily living and poor objective physical performance, with the strongest effects merging from the combination of hip and knee pain. These multivariable estimates have taken into account other potential covariates at initial Wave 2 such as body mass index, longstanding conditions, and pain treatment.

Our longitudinal results support other findings on the effects of pain on adverse outcomes. A US based study among older persons reported the impact of multi-site musculoskeletal pain, with two or three sites of pain associating with a $30 \%$ and $80 \%$ greater hazard for incident disability, respectively, compared with participants with no report on musculoskeletal pain (10). A longitudinal study conducted among Australian men aged 70 years and older reported that chronic knee pain is significantly associated with marked mobility disability, co-morbidity burden, and functional decline (9). A special feature of our study lies in the examination of both recurrent measures of exposures and repeat assessments across time. By using both subjective measure (self-reported ADLs and IADLs) and objective measure (physical performance during nurse visit), we confirm similar patterns and gradients of persistent general and specific pain on both adverse outcomes. 
Tests for trend of increasing exposure on the dose-response relationships were statistically significant at $p<0.05$ (data not shown).

In interpreting these findings, some limitations of the study should be noted. First, self-reported pain was used as the main exposure of interest. Since this is common among older adults, we used a threshold on the rating scale to define moderate/severe pain over the five-year period, rather than total pain reports. Second, since data are only collected in ELSA biennially, we were not able to take into account pain occurring between waves, and objective measures of physical performance were only available every 4 years. Third, there was no information whether the exact location of the pain or whether the pain occur in one of both sides of the knees or hips. Additional descriptive comparisons of respondents and non-respondents in 2004 showed that attrition was more common among older age groups (70+ years). However, this would be unlikely to affect the multivariate analyses.

By using prospective cohort data, we are able to measure the longitudinal impacts of recurrent and persistent pain among older adults. In particular, we captured gradients between site-specific pain and outcomes, which were strongest for the hip followed by the knee and back. Reports of hip and knee pain had the worst impact on future limitations in activities of daily living. Multiple-site pain requires more complex and specialised treatment than pain from a single site. Self-reported musculoskeletal pain is known to be a predictor of independence, unmet long-term needs for health care, and overall quality of life $(22,23)$. Our findings highlight the value of monitoring and management of both general and specific pain in order to promote sustained independence at older ages. 


\title{
Acknowledgments
}

The lead researcher in this study has been supported by the Australian Department of Education and Training (Endeavour Research Fellowship (5626_2016) and the Australian Research Council Centre of Excellence in Population Ageing Research (ARC CEPAR CE1101029).

\section{Disclosure statement}

\begin{abstract}
No potential conflicts of interest were disclosed.
\end{abstract}

\section{Reference}

1. Briggs AM, Cross MJ, Hoy DG, Sanchez-Riera L, Blyth FM, Woolf AD, et al. Musculoskeletal health conditions represent a global threat to healthy aging: A report for the 2015 World Health Organization World Report on Ageing and Health. Gerontologist. 2016;56 Suppl 2:S243-55.

2. Molton IR, Terrill AL. Overview of persistent pain in older adults. Am Psychol. 2014;69(2):197207.

3. Silva AG, Alvarelhao J, Queiros A, Rocha NP. Pain intensity is associated with self-reported disability for several domains of life in a sample of patients with musculoskeletal pain aged 50 or more. Disabil Health J. 2013;6(4):369-76.

4. March L, Smith EU, Hoy DG, Cross MJ, Sanchez-Riera L, Blyth F, et al. Burden of disability due to musculoskeletal (MSK) disorders. Best Pract Res Clin Rheumatol. 2014;28(3):353-66.

5. Cimmino MA, Ferrone C, Cutolo M. Epidemiology of chronic musculoskeletal pain. Best Pract Res Clin Rheumatol. 2011;25(2):173-83.

6. Nakamura M, Nishiwaki Y, Ushida T, Toyama Y. Prevalence and characteristics of chronic musculoskeletal pain in Japan. J Orthop Sci. 2011;16(4):424-32.

7. Brown ST, Kirkpatrick MK, Swanson MS, McKenzie IL. Pain experience of the elderly. Pain Manag Nurs. 2011;12(4):190-6.

8. Helme RD, Gibson SJ. Pain in older people. In: I.K. C, editor. Epidemiology of pain. Seattle: IASP Press; 1999. p. 103-12.

9. Fransen M, Su S, Harmer A, Blyth FM, Naganathan V, Sambrook P, et al. A longitudinal study of knee pain in older men: Concord Health and Ageing in Men Project. Age Ageing. 2014;43(2):206-12. 
10. Shah RC, Buchman AS, Boyle PA, Leurgans SE, Wilson RS, Andersson GB, et al. Musculoskeletal pain is associated with incident mobility disability in community-dwelling elders. J Gerontol A Biol Sci Med Sci. 2011;66(1):82-8.

11. Woo J, Leung J, Lau E. Prevalence and correlates of musculoskeletal pain in Chinese elderly and the impact on 4-year physical function and quality of life. Public Health. 2009;123(8):549-56.

12. Rogers NT, Marshall A, Roberts CH, Demakakos P, Steptoe A, Scholes S. Physical activity and trajectories of frailty among older adults: Evidence from the English Longitudinal Study of Ageing. PLoS One. 2017;12(2):e0170878.

13. Gale CR, Cooper C, Sayer AA. Prevalence of frailty and disability: findings from the English Longitudinal Study of Ageing. Age Ageing. 2015;44(1):162-5.

14. Melzer D, Gardener E, Guralnik JM. Mobility disability in the middle-aged: cross-sectional associations in the English Longitudinal Study of Ageing. Age Ageing. 2005;34(6):594-602.

15. Steptoe A, Breeze E, Banks J, Nazroo J. Cohort profile: the English Longitudinal Study of Ageing. Int J Epidemiol. 2013;42(6):1640-8.

16. Turner JA, Franklin G, Heagerty PJ, Wu R, Egan K, Fulton-Kehoe D, et al. The association between pain and disability. Pain. 2004;112(3):307-14.

17. Jones KR, Vojir CP, Hutt E, Fink R. Determining mild, moderate, and severe pain equivalency across pain-intensity tools in nursing home residents. J Rehabil Res Dev. 2007;44(2):305-14.

18. Chan KS, Kasper JD, Brandt J, Pezzin LE. Measurement equivalence in ADL and IADL difficulty across international surveys of aging: findings from the HRS, SHARE, and ELSA. J Gerontol B Psychol Sci Soc Sci. 2012;67(1):121-32.

19. Banks J, Nazroo J, Steptoe A, editors. The dynamics of ageing: evidence from the English Longitudinal Study of Ageing 2002-2012 London: Institute for Fiscal Studies; 2014.

20. Muriel A. ELSA's financial derived variables. Institute for Fiscal Studies, available at ifs.org.uk/elsa/user_day2011/muriel.pdf; 2011.

21. Lang IA, Llewellyn DJ, Alexander K, Melzer D. Obesity, physical function, and mortality in older adults. J Am Geriatr Soc. 2008;56(8):1474-8.

22. Hider SL, Whitehurst DG, Thomas E, Foster NE. Pain location matters: the impact of leg pain on health care use, work disability and quality of life in patients with low back pain. Eur Spine J. 2015;24(3):444-51.

23. Hartvigsen J, Davidsen M, Sogaard K, Roos EM, Hestbaek L. Self-reported musculoskeletal pain predicts long-term increase in general health care use: a population-based cohort study with 20-year follow-up. Scand J Public Health. 2014;42(7):698-704. 


\section{Figure Legends}

Figure 1 Study design flow diagram, English Longitudinal Study of Aeing (ELSA)

Figure 2 Prevalence of general and site-specific pain by age groups and sex, English Longitudinal Study of Ageing Wave 2 (2004) 
Table 1 Sociodemographic characteristics, English Longitudinal Study of Ageing (ELSA), Wave 22004

\begin{tabular}{|c|c|}
\hline Attributes & $\%(\mathrm{~N})$ \\
\hline \multicolumn{2}{|l|}{ Sex } \\
\hline Male & $56.3(2,818)$ \\
\hline Female & $43.7(2,192)$ \\
\hline \multicolumn{2}{|l|}{ Age } \\
\hline $50-59$ years & $35.6(1,795)$ \\
\hline 60-69 years & $26.7(1,840)$ \\
\hline 70-79 years & $21.9(1,099)$ \\
\hline $80+$ years & $5.7(286)$ \\
\hline \multicolumn{2}{|c|}{ Net total wealth quintiles } \\
\hline Quintile 1 & $17.2(849)$ \\
\hline Quintile 2 & $15.3(753)$ \\
\hline Quintile 3 & $19.9(982)$ \\
\hline Quintile 4 & $22.6(1,117)$ \\
\hline Quintile 5 & $25.0(1,231)$ \\
\hline \multicolumn{2}{|c|}{ Body mass index (BMI) } \\
\hline $\mathrm{BMI}<18.5$ & $3.9(198)$ \\
\hline BMI 18.5 to $<25$ & $23.6(1,182)$ \\
\hline BMI 25 to $<30$ & $39.7(1,988)$ \\
\hline BMI $30+$ & $32.8(1,642)$ \\
\hline \multicolumn{2}{|c|}{ Long-standing conditions } \\
\hline Yes & $54.3(2,719)$ \\
\hline \multicolumn{2}{|l|}{ Pain treatment } \\
\hline Yes & $7.5(378)$ \\
\hline
\end{tabular}


Table 2 General and site-specific pain on activities of daily living and physical performance, English Longitudinal Study of Ageing (ELSA)

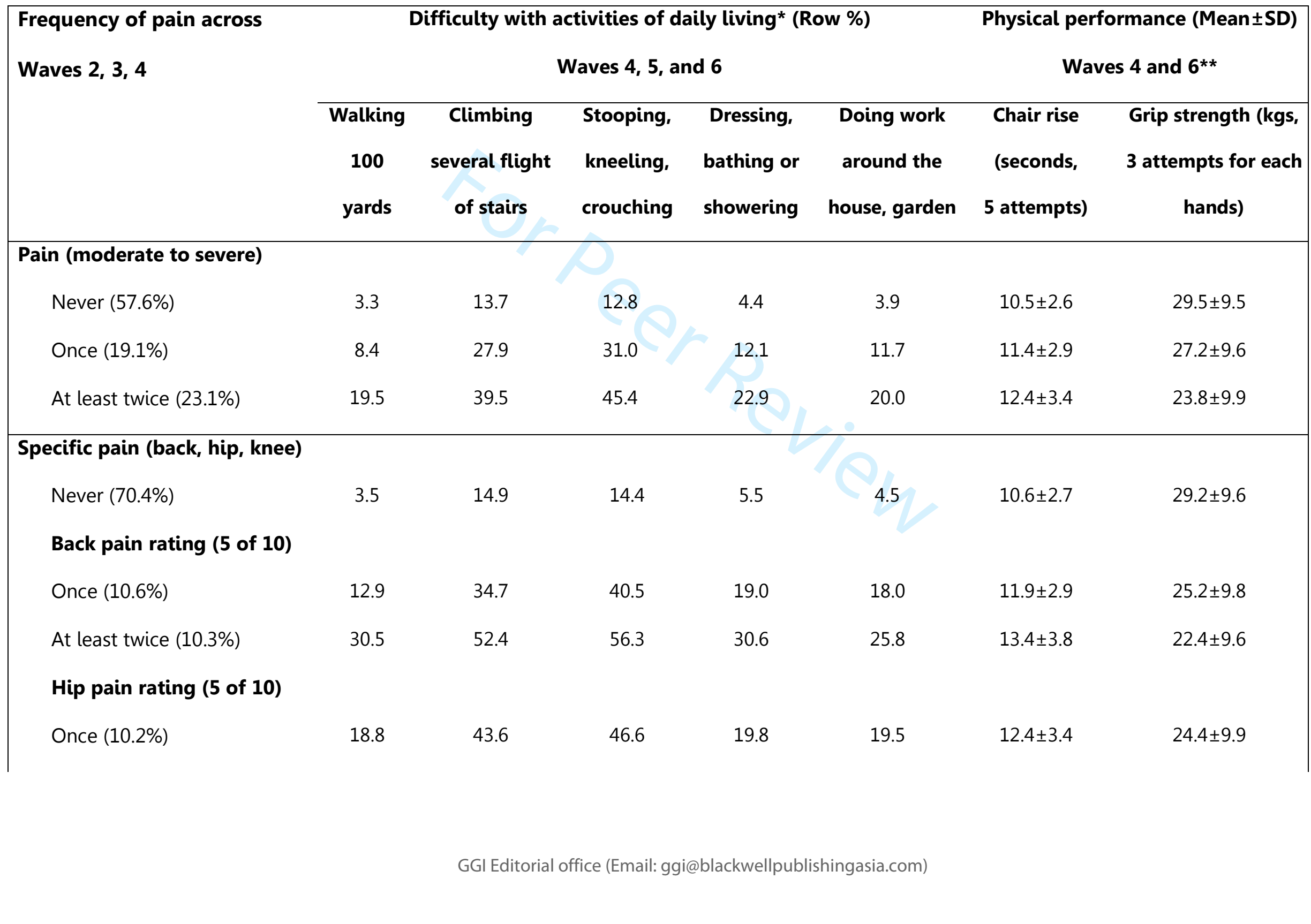




\begin{tabular}{|c|c|c|c|c|c|c|c|}
\hline At least twice (6.6\%) & 28.4 & 58.5 & 60.0 & 33.5 & 32.4 & $13.7 \pm 4.0$ & $22.5 \pm 9.8$ \\
\hline \multicolumn{8}{|c|}{ Knee pain rating (5 of 10 ) } \\
\hline Once (10.4\%) & 18.6 & 41.4 & 52.1 & 18.8 & 19.1 & $12.2 \pm 3.1$ & $25.0 \pm 10.1$ \\
\hline At least twice (9.9\%) & 29.2 & 57.0 & 74.1 & 31.2 & 29.4 & $13.4 \pm 3.8$ & $22.9 \pm 3.0$ \\
\hline \multicolumn{8}{|l|}{ ulti-site pain } \\
\hline \multicolumn{8}{|l|}{ Back and hip } \\
\hline Once (8.0\%) & 20.3 & 48.6 & 53.5 & 23.0 & 22.0 & $12.8 \pm 3.6$ & $23.5 \pm 9.6$ \\
\hline At least twice (4.9\%) & 28.4 & 56.8 & 59.4 & 37.3 & 33.9 & $13.8 \pm 7.9$ & $22.2 \pm 9.9$ \\
\hline \multicolumn{8}{|l|}{ Back and knee } \\
\hline Once (7.9\%) & 23.9 & 48.0 & 52.8 & 25.6 & 21.5 & $12.7 \pm 3.2$ & $24.4 \pm 4.3$ \\
\hline At least twice (5.6\%) & 35.8 & 55.3 & 81.4 & 39.8 & 35.5 & $13.9 \pm 4.1$ & $21.4 \pm 3.0$ \\
\hline \multicolumn{8}{|l|}{ Hip and knee } \\
\hline Once (8.1\%) & 23.8 & 48.3 & 56.4 & 23.4 & 22.3 & $12.9 \pm 3.7$ & $24.5 \pm 10.5$ \\
\hline At least twice (4.4\%) & 34.5 & 68.5 & 73.6 & 39.2 & 37.0 & $13.9 \pm 3.8$ & $21.5 \pm 10.0$ \\
\hline
\end{tabular}

*Reported at least twice across Waves 4, 5, 6

${ }^{* *}$ Nurse visit data were not available in Wave 5 
Table 3 Longitudinal analyses of general and site-specific pain on activities of daily living and physical performance, English Longitudinal Study of Ageing (ELSA)

\begin{tabular}{|c|c|c|c|c|}
\hline \multirow{3}{*}{$\begin{array}{l}\text { General and specific pain } \\
\text { reported at Waves 2, 3, } 4\end{array}$} & \multicolumn{2}{|c|}{ Multinomial odds ratios $[95 \% \mathrm{Cl}]$} & \multicolumn{2}{|c|}{ Mutivariable linear regression coefficient $[95 \% \mathrm{Cl}]$} \\
\hline & \multicolumn{2}{|c|}{ Number of limitations of daily activities* } & \multirow{2}{*}{$\begin{array}{c}\text { Chair rise, } 5 \text { attempts** } \\
\text { (seconds) }\end{array}$} & \multirow{2}{*}{$\begin{array}{c}\text { Grip strength, } 3 \text { times**; } \\
\text { (kilograms) }\end{array}$} \\
\hline & $0-1(\operatorname{Ref})$ & 4-5 & & \\
\hline \multicolumn{5}{|l|}{$\begin{array}{l}\text { Model } 1 \\
\text { Pain (moderate to severe) }\end{array}$} \\
\hline Once (19.1\%) & $1.56[1.21-2.01]$ & $2.31[1.60-3.34]$ & $\mathbf{0 . 3 1}[0.06,0.56]$ & $-0.18[-0.54,0.17]$ \\
\hline At least twice (23.1\%) & $1.77[1.42-2.20]$ & $2.48[1.70-3.61]$ & $\mathbf{0 . 7 4}[0.46,1.03]$ & $-\mathbf{0 . 8 7}[-1.25,-0.50]$ \\
\hline \multicolumn{5}{|l|}{$\begin{array}{l}\text { Model } 2 \\
\text { Back pain }\end{array}$} \\
\hline Once (10.6\%) & $1.47[1.12-1.94]$ & $2.21[1.96-4.52]$ & $0.37[0.04,0.70]$ & $-0.24[-0.68,0.21]$ \\
\hline At least twice (10.3\%) & $1.83[1.27-2.65]$ & $3.02[1.91-4.78]$ & $1.14[0.71,1.57]$ & $-\mathbf{0 . 7 3}[-1.23,-0.23]$ \\
\hline \multicolumn{5}{|l|}{ Hip pain } \\
\hline Once (10.2\%) & $1.91[1.43-2.56]$ & $\mathbf{3 . 1 8}[2.14-4.74]$ & $0.58\left[0.22_{L} 0.94\right]$ & $-0.19[-0.66,0.27]$ \\
\hline At least twice (6.6\%) & $2.51[1.55-4.07]$ & $4.26[2.42-4.79]$ & $1.47\left[0.93_{L} 2.02\right]$ & $-\mathbf{0 . 7 1}[-1.34,-0.08]$ \\
\hline \multicolumn{5}{|l|}{ Knee pain } \\
\hline Once $(7.3 \%)$ & $2.07[1.49-2.56]$ & 3.25 [2.21-4.89] & $\mathbf{0 . 3 9}[0.03,0.75]$ & $-0.33[-0.79,0.14]$ \\
\hline
\end{tabular}




\section{Model 3}

\section{Back and hip}

Once $(8.0 \%)$

1.86 [1.32-2.61]

2.93 [1.88-4.57]

$\mathbf{0 . 6 0}[0.19,1.01]$

$-0.40[-0.94,0.12]$

At least twice (4.9\%)

$2.35[1.34-4.12]$

$3.97[2.10-7.51]$

$1.66\left[1.01_{\llcorner} 2.32\right]$

$-0.79[-1.50,-0.07]$

\section{Back and knee}

Once (7.9\%)

2.04 [1.46-2.87]

2.19 [1.25-3.85]

3.51 [2.26-5.46]

$0.68[0.26,1.09]$

$-0.45[-1.14,0.23]$

At least twice (5.6\%)

4.19 [2.21-7.93]

$1.29[0.63,1.95]$

$-0.68[-1.20,-0.15]$

\section{Hip and knee}

Once (8.1\%)

2.08 [1.48-2.94]

3.92 [2.53-6.07]

$0.64[0.22,1.06]$

$-0.09[-0.61,0.44]$

At least twice (4.4\%)

3.24 [1.66-6.32]

5.16 [2.47-10.8] $1.47\left[0.70_{\perp} 2.24\right]$

*Adjusted for age, sex, wealth quintiles, body mass index, long standing conditions, limitations of daily living at Wave 2, pain treatment at Wave 2

${ }^{*}$ Adjusted for age, sex, wealth quintiles, body mass index, long standing conditions, chair rise scores at Wave 2, and pain treatment at Wave 2

${ }^{* * *}$ Adjusted for age, sex, wealth quintiles, body mass index, long standing conditions, grip strength scores at Wave 2 , and pain treatment at Wave 2 . 


\section{English Longitudinal Study of Ageing (ELSA)}

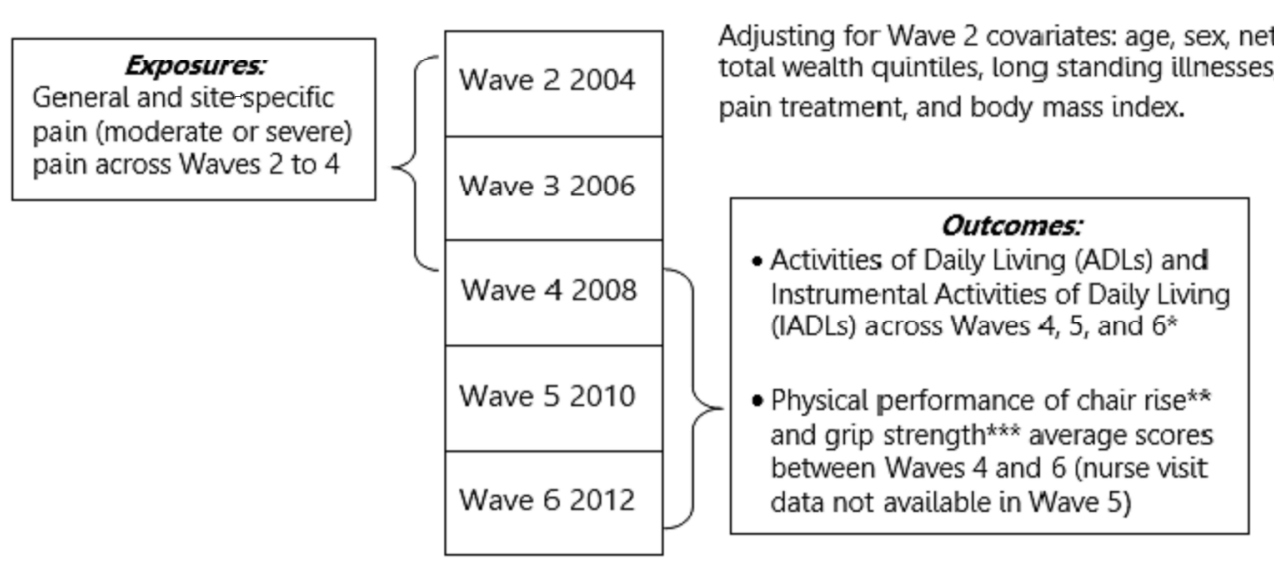

*Excluding individuals with impaired ADLs/IADLs in Wave 2

**Adjusting for Wave 2 initial chair rise performance scores

*** Adjusting for Wave 2 initial grip strength performance scores

Figure 1 Study design flow diagram, English Longitudinal Study of Aeing (ELSA)

$$
1455 \times 878 \mathrm{~mm}(96 \times 96 \mathrm{DPI})
$$




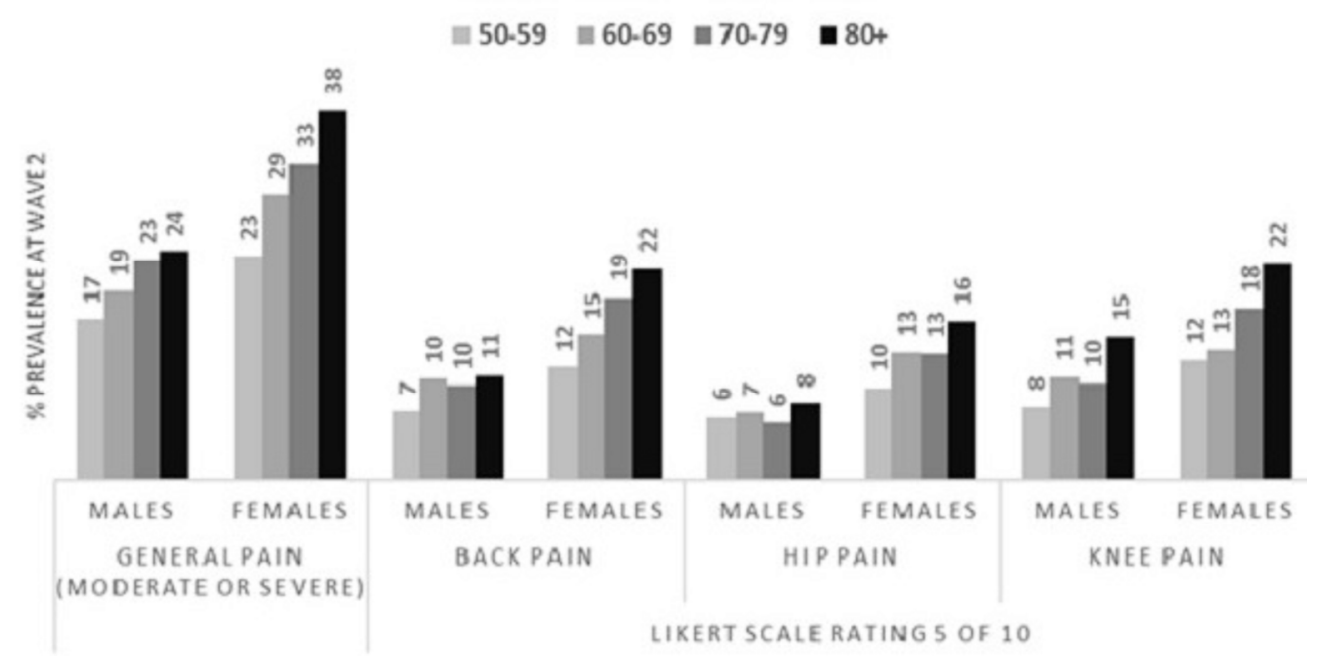

Figure 2 Prevalence of general and site-specific pain by age groups and sex, English Longitudinal Study of Ageing Wave 2 (2004)

$1455 \times 733 \mathrm{~mm}(96 \times 96 \mathrm{DPI})$

GGI Editorial office (Email: ggi@blackwellpublishingasia.com) 